\title{
Primary hyperparathyroidism
}

\section{Grzegorz J. Kowalski, Grzegorz Buła, Dominika Żądło, Agata Gawrychowska, Jacek Gawrychowski}

Department of General and Endocrine Surgery, Faculty of Medical Sciences in Zabrze, Medical University of Silesia, Katowice, Poland

\begin{abstract}
Primary hyperparathyroidism is an endocrine disorder that results in overproduction of parathyroid hormone by overactivated parathyroid gland leading to a significant rise in blood serum calcium. It results in hypercalcaemia, which has a significant impact mainly on the kidneys and bones and results in a variety of signs and symptoms. Primary hyperparathyroidism should be treated because, if left without any therapy, it can lead even to death. Surgery is considered as the best and only successful therapy, with very low risk of recurrence and relatively low complication rate. The aim of this review is to present clinical basis, aetiology, diagnostic possibilities, and treatment opportunities. (Endokrynol Pol 2020; 71 (3): 260-189)
\end{abstract}

Key words: primary parathyroidism; hypercalcaemia; parathyroidectomy; parathormone

\section{Introduction}

Primary hyperparathyroidism (PHPT) is a generalised endocrine disorder that leads to disturbances in the metabolism of calcium and phosphate due to an increase in autonomous production and secretion of parathyroid hormone by parathyroid glands. This excessive endocrine action usually results in hypercalcaemia and hypophosphataemia as well as significantly elevated concentration of PTH in serum is significantly elevated [1]. The prevalence of PHPT ranges between 0.1 and $1.0 \%$, which makes it one of the most common endocrine disorders, directly after diabetes and thyroid gland diseases [2, 3]. It affects significantly more females than males [1]. The overall incidence is approximately one case per 1000 per year in men over the age of 60 and two cases per 1000 in women over 60 years of age. There are several aetiologies of PHPT. In fact, many cases $(95 \%)$ are termed to be sporadic, where the most common underlying cause is solitary adenoma, which comprises about $84 \%$ of cases; double adenoma together with hyperplasia of all glands is present in approximately $15 \%$ of cases $[1,3]$. The remaining $1 \%$ of cases comprise very rare cases of parathyroid carcinoma [1]. Multiple endocrine neoplasia type 1 (MEN1) and type 2A (MEN2A) is responsible for around 5\% of all PHPT occurrence, which may occur in a familial pattern with no other endocrine abnormality already diagnosed. The aim of this work is to focus on and review the aetiology of PHPT. The clinical presentation of PHPT is highly vari- able. Multiple signs and symptoms may be presented by the patient, such as nephrolithiasis, peptic ulcers, and increased resorption of bone [1]. However, even in patients who have not developed any of these, screening tests such as blood calcium level raise awareness of the disease and lead to correct diagnosis. Due to such common incidence of this disease, there are already several known risk factors attributing to PHPT, such as lithium used to treat mental disorders over a prolonged period of time [1,2]. Additionally, those patients who were irradiated externally or internally with isotopes of iodine presented greater risks towards this disease $[1,4]$. Because tumours are responsible for most cases, the underlying cause of PHPT has to arise from abnormalities in genetics, more precisely from disturbances in the balance between proto-oncogenes, growth factor genes, and tumour suppressor genes. Is documented that sporadic and familial tumours are closely associated with defects in HPRT and MEN1 genes [64].

\section{Aetiology}

Solitary adenomas are responsible for approximately $85 \%$ of all cases of PHPT. In $15 \%$ of them, hyperplasia affects all glands with the main predisposition to chief cells. In very rare cases, adenomas may be found in more than one gland, with preservation of other, healthy glands [1]. However, in each case, resection of the affected gland is the best form of curing the disease. A normal parathyroid gland is the size of 
an apple seed and weighs approximately $0.5 \mathrm{~g}$. Microadenomas are defined as tumours that weigh less than less than $0.1 \mathrm{~g}$, while a giant adenoma is over 2 g. Adenomas usually are found within inferior parathyroid glands and are up to $1 \mathrm{~g}$ in weight, but there are cases in which the neoplasm reaches the weight of a normal healthy parathyroid gland, i.e. $25 \mathrm{~g}$ [1, $59,60]$. The establishment of distinction between hyperplasia and adenoma may be problematic. Where there is hyperplasia, the glands that are involved are sometimes so enlarged and asymmetric that they are normal in gross appearance. In such cases, a biopsy taken by the surgeon during the surgery is essential for interpreting findings and gross interpretation, because microscopic histological examination divulges the uniform pattern of chief cells and disappearance of fat [1]. If adenoma is present, there are normal glands present with physiological distribution and content of all the cells as well as fat [1]. Parathyroid carcinoma accounts for around 1\% of PHPT cases and is usually not considered to be aggressive. If resection of the entire gland without rupture of the capsule is performed as soon as possible, long-term survival without reccurrence is very likely, but still there are cases of relapse. On the other hand, even if the recrudescent parathyroid gland carcinoma grows, it is a very slow process that usually spreads within the neck. In such cases, again surgical resection may be manageable. Such a carcinoma may be aggressive and is likely to form metastases in lung, liver, and bones, which may be found during the initial operation [1]. However, it is hard for a surgeon to decide if the tumour is actually carcinoma and to eventually distinguish it from other forms of primary hyperparathyroidism. The potential diagnostic clue is measuring the degree of calcium elevation, where, in the case of carcinoma, it equals around $14 \mathrm{mg}$.

Hyperparathyroidism can occur in patients without other endocrine disease in a familial pattern. There are few syndromes of multiple endocrine neoplasia (MEN), predominantly type 1 and type 2 . Type 1 disorder (MEN1) is also called Wermer's syndrome and appears as hyperparathyroidism together with tumours of the pituitary gland or pancreas. It may also be connected with Zollinger-Ellison syndrome due to the occurrence of peptic ulcers and gastric acid over secretion in such patients [1]. Type 2 disorder (MEN2) is another endocrine constellation, with autosomal dominant pattern of inheritance, and is closely associated with medullary carcinoma of the thyroid as well as with pheochromocytoma. Tumours associated with the type of multiple endocrine neoplasia are useful for distinction because pituitary gland or pancreatic tumours will not appear in MEN2, e.g. tumours of thyroid and adrenal medulla are not found in MEN1 [1].

\section{Signs and symptoms}

Currently, half or more patients with hyperparathyroidism are asymptomatic, and specific signs and symptoms occur primarily in the kidneys in the form of stones, as well as bone diseases. Nowadays the frequency of detection of asymptomatic cases has increased, mainly due to routine biochemical tests done for other purposes, so the incidence of renal complications is lower. Normal cellular function depends on calcium homeostasis, and in PHPT it is disrupted. This results in manifestations such as urinary, musculoskeletal, gastrointestinal, cardiovascular, neuromuscular, and neuropsychiatric symptoms [1]. The kidney involvement is mainly due to deposits of calcium, leading to nephrolithiasis in renal parenchyma and hypercalciuria [5]. Renal stones are mainly composed of calcium oxalate and calcium phosphate and may lead to urinary tract obstruction or infection, which results in loss of physiological renal functions. Nephrolithiasis is found in about $20 \%$ of PHPT cases, and its number is still increasing [6]. In increased parathormone production, calcium, which needs to be filtered, exceeds the renal tubules capacity of possible reabsorption and results in hypercalciuria in about $40 \%$ of patients [3]. On the other hand, nephrocalcinosis leads to phosphate retention and results in a significant decrease of urinary system functions. Usually it is hard to find both conditions in the same patient, so diagnosis must be made carefully. Hypercalcaemia manifests in the musculoskeletal system. There may also be a direct effect of PTH on bones because this hormone acts on osteoblast receptors, stimulating them to differentiate into osteoclasts, and hence leading to cortical bone resorption [7]. If the level of parathormone is chronically increased, it causes osteoporosis, osteopaenia, or fibrosis [5]. The unique form of bone involvement in hyperparathyroidism is osteitis fibrosa cystica, which has several specific histological findings in microscopic examination, such as a reduction in the number of trabeculae and an increase in the amount of giant multinucleated osteoclasts on the bone surface. Fibrous tissues that have replaced physiological bone marrow elements can also be observed. Because osteoporosis and osteopaenia are understood as the most common diseases closely related to PHPT, osteitis fibrosa cystica is less common, and its reduced frequency has been not yet explained [1]. Manifestations of PHPT in CNS vary from moderate disturbances of personality to very severe mental disorders, including consciousness impairment, e.g. coma. Many patients suffering from PHPT have nonspecific symptoms including fatigue, 
disorders of circadian rhythm, mood depression, inability to focus, concentrate, and memorise, and a decrease in creativity. It is so general that it cannot be considered as a clear indication for parathyroidectomy, especially because the third workshop from 2008 showed that in PHPT the presentation of psychological disorders is quite common, but the precise extent of neuropsychiatric symptoms is not clear [1,8]. Neuromuscular disorders in PHPT are directly connected with muscle weakness (especially peripheral), general fatigue, and marked muscle atrophy. Hence, the electromyogram tends to be within normal range and atrophic muscle fibres are without pathological changes. After surgical excision of parathyroid glands, atrophy may be totally reversed without any longer striking [1]. The group of subtle manifestations of PHPT includes gastrointestinal problems such as abdominal pain, and stomach and pancreas function disorders [1]. In addition, patients complain of nausea, vomiting, and constipation, and there are rare cases of acute pancreatitis [9]. There is also a marked increased tendency of duodenal ulcer occurrence. In patients suffering from MEN1 type of hyperparathyroidism, duodenal ulcers are resulting from associated tumours of the pancreas that are actively secreting increased amounts of gastrin [1]. In addition to these main findings, there have been recent studies showing a direct connection between PHPT and the cardiovascular system, such as calcification of valves and certain areas of myocardium. Moreover, lipid abnormalities resulting in arterial hypertension and coronary artery disease, hypertrophy of left ventricle, and disturbances of conduction also occur [6]. In some cases, patients with PHPT present a parathyroid crisis because of increased concentration of calcium in serum. They are significantly dehydrated, which leads to an even greater increase in blood calcium levels. Such a condition is so dangerous because patients may have manifest disruption of cardiac and kidney functions, deterioration of central nervous system (CNS), and gastrointestinal disturbances in the form of nausea and vomiting connected with abdominal pain [10].

\section{Diagnosis}

\section{Proper anamnesis}

The first step in indicating the disease is to form a differential diagnosis, which is made of: PHPT, lithium drug therapy, malignancies, increased calcium intake or reduction in excretion, excess of vitamin $\mathrm{D}$, long immobilisation of the patient, hyperthyroidism, and multiple myeloma [11]. As neoplasm or malignancy stands for the most common cause of hypercalcaemia, PHPT is highly probable [12]. In such conditions, hypercalcaemia associated with PHPT is related to suppressed
PTH levels because the negative feedback mechanism is still intact in parathyroid glands [5]. At the beginning of the diagnostic process of PHPT it is highly relevant to collect the family history with a focus on the clinical grounds described previously. The correct anamnesis can give a chance to confirm or exclude PHPT forms which are hereditary, particularly MEN 1 and 2 types. If the family history of PHPT is positive, genetic testing should be considered, especially in the diagnosis of patients younger than 50 years old [13]. After the family anamnesis, the drug history should be made with emphasis on past or current usage of medicaments such as diuretics, which can raise the calcium level in blood serum without PHPT, as well as lithium, which is used to treat mental disorders such as depression. It is relevant because long-term ingestion of those substances is thought to be closely associated with development of PHPT [1, 13].

\section{Biochemical testing}

The biochemical tests are very useful in the diagnosis of PHPT because they allow one to mark the hypercalcaemia that is caused by increased secretion of PTH from parathyroid glands [1]. For this aim, immunoassays are also used, but there are problems occurring with the interpretation of such results. Characteristically, the immunoreactive PTH level can be elevated or inappropriately normal [1]. The diagnosis is classical if the calcium is elevated when the concentration of PTH is also increased [13]. Sometimes it happens that mild PHPT occurs with PTH or calcium in blood serum is normal by laboratory means, so there appears a need for comparison of both values together, which will lead to a biochemically abnormal result [5]. The PHPT is called normal calcaemic when PTH is high and serum calcium level seems to be normal. However, inappropriate secretion of PTH is found in patients with hypercalcaemia and inappropriately normal or unsuppressed PTH level [1]. Physiologically, when hypercalcaemia occurs, it should result in the activation of a negative feedback process acting directly on parathyroid glands, leading to suppression of PTH secretion. That implies that PHPT should be diagnosed even with normal levels of PTH in the serum when hypercalcaemia was detected. The comparison of found values is summarised in Table 1 . There are some different reported opinions explaining the normocalcaemic variety of PHPT, showing that some surgeons assume that it may be a classical PHPT caused by disturbances in PTH secretion regulation, while others surmise the resistance to the action of this hormone [14].

Hypercalciuria is often found in hyperparathyroidism; nevertheless, parathormone decreases calcium clearance so that excretion of calcium in urine (daily) 
Table 1. Clinical presentation in hyperparathyroidism. Based on [66]

\begin{tabular}{lcc}
\hline Classical presentation & Calcium & PTH \\
\hline Classical PHPT & Increased & Increased \\
\hline $\begin{array}{l}\text { Inappropriate secretion } \\
\text { of PTH }\end{array}$ & Increased & $\begin{array}{c}\text { Within normal } \\
\text { range }\end{array}$ \\
\hline Normocalcaemic PHPT & $\begin{array}{c}\text { Within normal } \\
\text { range }\end{array}$ & Increased \\
\hline
\end{tabular}

PTH — parathormone; PHTP — primary hyperparathyroidism

is lower than in patients in which hypercalcaemia was found, which was caused by a reason other than parathyroid gland disorders [1]. Primary hyperparathyroidism is not only associated with disorders of calcium metabolism; hence, in blood serum of patients, the phosphate level is usually low. However, there are cases of patients with already developed renal failure and normal phosphate levels. Hypophosphataemia is not as significant a diagnostic measure as hypercalcaemia because levels of phosphates are influenced by daily dietary intake and variations in diuresis, so the best time for sampling is in the morning. Nevertheless, patients who are suffering from severe hypercalcaemia may have decreased blood serum phosphate level (1). Assessing the response of blood serum calcium to administration of glucocorticoid drugs is a useful diagnostic criterion. It allows to differentiate the hypercalcaemia in PHPT from hypercalcaemia associated with other diseases, among which are: sarcoidosis, vitamin D intoxication, multiple myeloma, neoplasms, and carcinomas. In hyperparathyroidism, administration of $100 \mathrm{mg}$ per day for around 10 days do not result in lowering the calcium levels unlike in other diseases related to hypercalcemia [1]. There are many tests to establish the function of parathyroid glands. Those are based on the effects of this hormone on renal action with phosphate; in particular, parathormone successfully decreases renal phosphate resorption. The clearance of phosphate can be determined by a standard technique within one- and two-hour intervals. Normal clearance of phosphate is about $10.8 \mathrm{ml}$ per minute, while if the value reaches $50 \%$ to $60 \%$, it may indicate hyperparathyroidism, compared to normal values of phosphate resorption, where it reaches about $80 \%$. Moreover, the possibility to measure nephrogenous cAMP is also appropriable in the diagnostics of PHPT [1].

\section{Molecular testing}

Every neoplastic change within the organism results from disturbance of equilibrium maintenance between proto-oncogenes and tumour suppressor genes. Additionally, several other defective transmission mechanisms have been described, such as cell cycle regulation or apoptosis induction [15]. The vast majority of primary hyperparathyroidism cases are caused by hyperplasia and further differentiation of modified cells, so genetic laboratory testing, which is more and more available, plays an essential role in prevention, diagnostics, and classification of tumours. Sporadic PHPT, as mentioned before, stands for up to $95 \%$ of all PHPT cases. Although it is so widespread, the exact causative genetic mutations are not well understood. Nevertheless, in sporadic PHPT, there are two genes closely linked to parathyroid adenomas such as: oncosuppressor gene MEN1 and cyclin D1 gene, which are located on the $11^{\text {th }}$ chromosome. In around $30 \%$ of sporadic parathyroid tumours, the homozygous loss of MEN1 locus has been found, so it is the major genetic somatic cause in sporadic PHPT [16]. On the other hand, overexpression of cyclin D1 has been associated with up to $40 \%$ of sporadic adenomas and around $90 \%$ of carcinomas [17]. Recently, a study confirmed MEN1 mutation and evaluated additional HRPT2 gene mutation associated with development of large parathyroid adenoma (LPA). In the majority of tumours, the observation brought total or partial loss of parafibromin expression, and in some of them loss of APC gene expression was found [18]. Negative parafibromin staining indicated a higher risk of recurrence/metastasis and mortality. The immunohistochemical staining of parafibromin seems to be more promising in predicting outcomes for patients with parathyroid carcinoma. Ki-67 antigen often correlates with the course of cancer, which is also of great prognostic value in these diseases. Positive response to the presence of Ki-67 is more common in parathyroid cancer compared to benign lesions [61]. Nevertheless, cases taken into consideration were a result of the Array Comparative Genomic Hybridisation (aCGH) method, which has showed recurrent copy number alterations most frequently involving loss in $1 \mathrm{p}(29 \%)$, gain in 5 $(38 \%)$, and loss in 11q (33\%). Another study points out that while bi-allelic inactivation of CDC73/HRPT2 seems unique to parathyroid malignancy, aberrant activation of cyclin D1 and Wnt/-catenin signalling has been reported in benign and malignant parathyroid tumours (19). A different study [20] showed the importance of parathormone gene mRNA studying. All of the investigated cases were positive by PTH-PCR, which was confirmed by histological investigation - it led to the conclusion that reverse transcriptase-polymerase chain reaction (RT-PCR) detection of parathormone $(\mathrm{PTH})$ gene mRNA in needle aspirates is a very useful method for parathyroid adenoma diagnosis. Parathyroid carcinoma is one of the most malignant neoplastic changes of parathyroid glands; thus, the molecular changes have been studied in order to better understand the disease itself and to create the best therapy. A study 
(21) evaluated tumour-specific genetic changes using next-generation sequencing (NGS), resulting in the conclusion that the most common genetic alterations were found within two pathways: the PI3K and TP53 pathways, which leads to the conclusion that this method is also very helpful, and more studies based on those pathways should be taken into consideration. Molecular allelotyping was performed and described in our recent study. Polymorphic microsatellite markers were used, leading to the conclusion that parathyroid carcinomas develop de novo rather than from previously existing parathyroid adenomas [22]. Mutations that cause to primary hyperparathyroidism should be detected if multiple endocrine neoplasia type 1 (MEN 1) is considered as the diagnosis. This disease is autosomal dominant in its inheritance pattern. The responsible mutation occurs in oncosuppressor gene MEN1 encoding protein menin, which negatively regulates progression of cell cycle, controls integrity of DNA, and partially regulates the process of apoptosis. In advanced diagnostics, the MEN1 test is advised. The positive result leads to the performance of a thymectomy with parathyroid intervention as a prophylaxis in order to prevent the thymic carcinoids. If the MEN1 test is negative, surgical intervention is needed but restricted to parathyroid glands that are hyperplastic or adenomatous [16]. Multiple endocrine neoplasia type 2 and genetic mutation leading to this disorder have also been described in different studies. Among the two variants, MEN2A is responsible for up to $80 \%$ of MEN2 syndromes. MEN2A syndrome is composed of hyperparathyroidism, hyperplasia of parathyroids, pheochromocytomas and medullary carcinoma [23]. Within those patients PHPT occurs in around $25 \%$ of MEN2A cases, usually after 30 years of life, and usually without any clinical symptoms. PHPT that is related to MEN2A may range from single parathyroid gland adenoma to multiglandular hyperplastic changes [16]. MEN2 syndrome is inherited in an autosomal dominant pattern [24]. The proto-oncogene RET is the one susceptible gene associated with MEN2, located on chromosome 10. The RET gene encodes membrane tyrosine kinase receptor protein consisting of three portions: extracellular, transmembrane, and intracellular domains. The extracellular portion is composed of four domains and is responsible for calcium binding, while intracellular portion consists of two distinct tyrosine kinase domains. Only one allele of the RET gene needs to be mutated in order to develop MEN2 syndrome. Morbidity in MEN2 syndrome occurs mostly because of chronic hypercalciuria, and to prevent this, a test may be performed. Nevertheless, PHPT in MEN2A is usually without clinical symptoms; prophylactic parathyroidectomy is not recommended [16].
Single immunohistochemical markers such as parafibromin and Ki-67 are among the most studied and most utilised, but many additional markers have been, and continue to be, evaluated, such as galectin-3, PGP9.5, $\mathrm{Rb}$, bcl2, p27, hTERT, mdm2, and APC. Although not widely available in many laboratories, a panel of immunohistochemical markers may prove most useful as an adjunct in the evaluation of challenging parathyroid tumours [62].

\section{Imaging methods}

The use of imaging before performing surgery for patients with hyperparathyroidism is a golden standard in order to locate hyperfunctioning glands and determine its effect on the whole organism. Usually a single, adenomatous, and enlarged gland is the cause of primary hyperparathyroidism, so a pre-operative work-up should be performed because it will help the surgeon to plan the best surgical approach to the patient and it serves as a helpful guide for choosing the type of operation [25]. The preoperative diagnostic studies may be non-invasive or invasive. Within non-invasive methods the following can be distinguished: neck ultrasound (US), single photon emission computed tomography (SPECT), Tc-99m sestamibi imaging, magnetic resonance imaging (MRI), four-dimensional CT scan (4-D CT), and positron emission tomography combined with CT scan (Tab. 1). Neck ultrasonography is a type of functional imaging method that can be used any time before or during surgery due to its low cost and good access. It enables quick localisation of the affected gland, thus decreasing the extent of neck dissection $[26,27]$. Subtraction scintigraphy Tc-99 sestamibi is also widely used. The latest research shows that it is essential for localisation of rapid washout parathyroid adenomas. It also showed that pre-operative PTH levels and the size of the gland where major determinants of Tc-99 sestamibi positivity on early-phase Tc- $99 \mathrm{~m}$ scans, and size is an independent factor of late-phase Tc-99m [28, 29]. There is a possibility to combine neck ultrasonography and Tc-99m sestamibi in order to increase the specificity and sensitivity to over $90 \%$ [28]. If both methods are positive, the exact surgical approach may be determined. On the other hand, it those are negative, most surgeons decide to identify all glands during an open parathyroidectomy because cases of false negative scintigraphy, in patients with primary parathyroidism, are quite rare and usually correspond to lesions beyond the equipment's resolution limit [29]. Subsequent imaging methods are $4 \mathrm{D}-\mathrm{CT}$ scan and MRI, as more sophisticated and expensive radiological methods that can be helpful in discovering ectopic parathyroid glands. In most cases those methods are used in reop- 
Table 2. Imaging studies. Based on [66]

\begin{tabular}{|c|c|c|c|c|}
\hline Image name & Sensitivity (\%) & Specificity (\%) & Main advantages & Main disadvantages \\
\hline \multirow{3}{*}{ Tc-99m } & \multirow{3}{*}{$80-90$} & \multirow{3}{*}{$78-98$} & \multirow{3}{*}{ Minimal radiation } & Limited sensitivity if more glands than one are affected \\
\hline & & & & Takes quite a long time \\
\hline & & & & $\begin{array}{l}\text { Does not assuredly distinguish thyroid nodule } \\
\text { from parathyroid glands }\end{array}$ \\
\hline \multirow{4}{*}{$\begin{array}{l}\text { Neck } \\
\text { ultrasound }\end{array}$} & \multirow{4}{*}{$70-80$} & \multirow{4}{*}{$80-89$} & \multirow{4}{*}{$\begin{array}{l}\text { Quick and cheap } \\
\text { No radiation } \\
\text { May be performed before } \\
\text { or during surgery }\end{array}$} & \\
\hline & & & & \\
\hline & & & & Low sensitivity in substernal, retrotracheal, \\
\hline & & & & and retroesophageal glands \\
\hline \multirow{3}{*}{$\begin{array}{l}\text { 4-dimensional } \\
\text { CT scan }\end{array}$} & \multirow{3}{*}{$60-80$} & \multirow{3}{*}{$85-98$} & \multirow{3}{*}{$\begin{array}{l}\text { More sensitive for } \\
\text { smaller lesions than neck } \\
\text { ultrasound }\end{array}$} & Exposure to radiation \\
\hline & & & & Involves risk of application due to contrast agent \\
\hline & & & & $\begin{array}{l}\text { Not able to differentiate parathyroid glands from, } \\
\text { for example, lymph node tissue }\end{array}$ \\
\hline MRI & $70-80$ & $90-95$ & No radiation & $\begin{array}{l}\text { Availability is very limited due to cost, time consumption, } \\
\text { patients' maintenance of metal objects inside the organism, } \\
\text { and patients' claustrophobia }\end{array}$ \\
\hline
\end{tabular}

Tc-99m — Tc-99m sestamibi imaging; US — ultrasound; 4-DCT — 4-dimensional computed tomography; MRI — magnetic resonance imaging

erative cases [30] and are helpful in early distinguishing of parathyroid carcinomas from other parathyroid lesions. Recent research showed that CT features of high short-to-long axis ratio, irregular shape, and the presence of peritumoral infiltration and calcification may be indicators for differentiating parathyroid carcinoma from benign parathyroid hyperplasia [31]. Radiation exposure to neck by $4 \mathrm{D}-\mathrm{CT}$ is relatively high and its usage is limited to first investigation in order to diagnose of primary parathyroidism. Exposure to radiation should be restricted if possible, so evaluation of the percentage arterial enhancement as objective radiological index can be helpful, and used to determine parathyroid lesions (adenoma/hyperplasia) and discriminate it from thyroid tissue and lymph nodes [32]. Positron emission and computed tomography (PET/CT) may also be performed as a non-invasive diagnostic method. Parathyroid adenoma and probably most parathyroid hyperplasia may be exactly localised in patients with primary hyperparathyroidism using choline PET/CT. Thus, minimally invasive surgical intervention may be further performed with lower risk of complications [33, 34]. Ultrasound, Tc-99m, and SPECT/CT all have greater value in the diagnosis of parathyroid adenoma than parathyroid hyperplasia. Tc-99m and SPECT/CT should be optimal for detecting parathyroid adenoma, where early scanning is better than delayed scanning. In comparison, ultrasound is slightly better in the localisation of parathyroid hyperplastic lesions than Tc-99m and SPECT/CT. The best way to improve accuracy in localisation of parathyroid hyperplastic lesions is to combine ultrasonography with SPECT/CT or Tc-99m. This should be taken into consideration as a first-line diagnostic method when detecting parathyroid hyperplasia [35].

One of the most promising imaging techniques in primary hyperparathyroidism is PET/CT with choline-based tracers. Choline PET/CT shows favourable results in the detection of hyperfunctioning parathyroid tissue and may replace conventional technetium-99m-sestamibi scintigraphy using planar dual-phase imaging, SPECT, or SPECT/CT in preoperative planning of parathyroid surgery [63]. Localisation of single gland adenomas can be achieved with choline PET/CT in $80-96 \%$ of cases. The high sensitivity and accuracy of choline PET/CT imaging is documented even in cases of repeated surgery for recurrent PHPT, in coexistent nodular goitre, or in the detection of adenoma in atypical localisation [65].

Invasive methods are used usually reoperative cases where anatomical localisation of the affected gland is even more crucial and the following are distinguished: selective arteriography, fine needle aspiration (FNA), and selective venous sampling [36]. Selective arteriography is useful because all parathyroid glands that are enlarged are highly vascular. Because of this fact, the contrast may be easily injected and visualise the affected glands very well. It can identify even small and ectopic parathyroid glands and is commonly used in combination with venous sampling methods, where thyroid venous plexus and thymic veins are required. This technique brings essential differentiation between hyperplasia, which exposes increased PTH within venous plexus bilaterally, and adenomas, which produce only unilateral elevated secretion of PTH [37]. All invasive methods are involved in higher risk of complications 
than non-invasive methods. A suspicious tissue seen on imaging can also be sampled by FNA. After aspiration, the content needs to be tested for PTH level. This method is not needed for conclusive diagnosis in most cases. Nevertheless, it may be helpful when the area of suspicion is not characteristic and is usual for parathyroid glands such as areas lateral to the carotid artery. All invasive methods involve a higher risk of complications than non-invasive methods.

\section{Treatment}

Surgical treatment is considered as the only suitable therapy for PHPT. Parathyroidectomy is performed for symptomatic as well as for asymptomatic patients. Exploration of parathyroid glands should be performed by an experienced surgeon, preferably with the help of a pathomorphologist. It should be taken into consideration that several clinical signs are helpful in predicting the existing pathology [1]. During operation, some critical decisions regarding further management need to be made, so the examination of removed tissue should be examined in order to direct the further course of surgery. Regarding the decision of whether the operation is irreplaceable, consensus guidelines exist; recent ones recommend operation for asymptomatic patients with PHPT, who are younger than 50 years of age, having the following: signs of bone disorders such as osteoporosis, blood serum calcium level reaching $1 \mathrm{mg}$ or more than upper acceptable limits of normal value, creatine clearance should be lower than $60 \mathrm{cc}$ per minute, or nephrolithiasis. There are also asymptomatic cases with worsening laboratory findings, which should also be admitted for surgery according to the procedure established during the third international workshop by the International Task Force on PHPT [38]. Usually, medical follow-up and performing a lot of diagnostic tests are quite expensive and prolonged in time, unlike surgery, which is more effective for asymptomatic and even more for symptomatic patients [38]. Nowadays, such surgeries are performed by experienced endocrine surgeons, who perform an increasing number of operations. Statistically, the risk of such operations is relatively low because people under 60 years old better undergo anaesthesia than prolonged drug therapy [1].

\section{Bilateral neck exploration}

Bilateral neck exploration is a traditional method of primary hyperparathyroidism treatment and has been a gold standard for decades. Here, endotracheal intubation is required followed by identification of all parathyroid glands by exploration of the central part of the neck. During this procedure, the surgeon is responsible for careful judgement of the tissue, which is grossly changed in appearance, and finally its removal. The exposure and incision are very similar to those used in standard thyroidectomy. The crucial step is performing the division in the middle thyroid veins, mobilisation of the thyroid lobes, and identification of the thyroid artery together with the recurrent laryngeal nerve, followed by their retraction by vascular slings. Afterwards, without or with incision of the fascia, the superior parathyroid gland is slated. In most cases, the upper parathyroid glands are related to the inferior thyroid artery, and the inferior parathyroid glands recline within $2 \mathrm{~cm}$ radius of the lower pole of the thyroid gland. Then, the inspection of the thyrothymic ligament and tracheoesophageal groove is required in case of occurrence of ectopic parathyroid glands. Ectopic glands can rarely also be found within the thyroid gland and carotid sheath, so those should also be checked. If all steps are accomplished, the surgeon may move on to performing the transcervical thymectomy. Transcervical thymectomy starts with localising the adenoma, followed by its ligation and division of the pedicles. Once it is done, it should be cleared from the surrounding tissue and removed. The diagnosis is usually confirmed by frozen section because differentiation between hyperplasia and adenoma is not an easy task. When the adenoma is excised, the surgeon identifies the remaining parathyroid glands or the ipsilateral gland in order to perform biopsy or frozen section investigation. If the results are normal, those glands do not require further procedure. A subtotal 3.5 parathyroidectomy is recommended in the case of multi-glandular hyperplasia [48]. The Bilateral Neck Exploration technique enables the identification of all four parathyroid glands and further resection of the most enlarged and investigation of glands that seem not to be changed in gross appearance. The main disadvantages of this method are long operative time and prolonged post-operative hospitalisation period in comparison to less invasive ones. The cure rate reaches $98 \%$, and the morbidity is relatively low, which makes this procedure a golden standard in the therapy of the underlying cause of primary hyperparathyroidism.

\section{Unilateral neck exploration}

This technique enables a less invasive surgical approach for the treatment of the primary hyperparathyroidism. It has many advantages such as low risk for recurrent or persistent hyperparathyroidism and the ability to use local anaesthesia [39]. Unilateral neck exploration brings the same results as bilateral exploration but has a better cosmetic result and shorter operative time. On the other hand, there are several disadvantages of this technique, such as not being dependable in multi-glandular disease, where this approach should be taken into consideration 
as having potential to fail [40]. This method is also restricted to correct localisation, so imaging methods with improved modalities in order to enhance the sensitivity in localisation of adenoma are used. Usage of rapid intra-operative intact parathyroid hormone (iPTH) assays are helpful in the confirmation the success of the operation when the patient is still on the table in the operating room [41] but still has almost $50 \%$ false positive results in patients with double adenoma. That may lead a surgeon to stop the procedure even if they should continue. On the other hand, $13 \%$ of false negative results may guide the doctor to perform often extended resections when there is no affected gland left. This leads to the conclusion that currently there is no possibility to be sure that all unexplored glands are not affected, but still Unilateral Neck Exploration has some essential advantages [42]. The solution to cover diagnostic demands is proper pre-operative testing. The technique itself is not very time consuming. The patient needs to be placed in a supine position and has to have an extended head with arms by the body's side. After usage of local or general anaesthetics, a $2 \mathrm{~cm}$ transverse incision takes place above the sternal notch in a skin crease. It should be done over the expected position of the parathyroid adenoma and the sternocleidomastoid muscle. Then, the strap muscles are dissected and separated. The thyroid lobe needs to be gently retracted superiorly. The affected, enlarged parathyroid is dissected with or without identification of recurrent laryngeal nerve. Afterwards, absorbable sutures are used to close the wound [41].

\section{Minimally invasive parathyroidectomy and minimally invasive radio-guided parathyroidectomy}

Minimally invasive parathyroidectomy is used together with a pre-operative localisation test, which suggests a unilateral approach. This technique is normally performed under cervical block, and for success determination of intra-operative iPTH is used [43]. There is a documented procedure of excision of even giant parathyroid adenoma using minimally invasive transcervical approach after its radiological localisation pre-operatively resulting in full recovery. This case leads to the conclusion that this method is an advantageous alternative for neck exploration or sternotomy [44].

Minimally invasive radio-guided parathyroidectomy is performed with exploitation of a gamma camera, which enables usage of increased sensitivity of Tc-99m sestamibi intra-operatively [45]. This method facilitates establishment of exact localisation of the affected gland and following its removal. Patients with a positive pre-operative Tc-99m sestamibi can be operated without general anaesthesia, which is an advantage of minimally invasive surgery [43]. This technique requires usage of a sestamibi scan (MIBI scan). If the MIBI scan is positive and shows a single pathological focus within the neck area, it is required to perform MIRP. Following this procedure, the patient needs to be re-injected up to 60 minutes before operation [41]. Afterwards, anaesthesia (local or general) should be administered and a 2-4 $\mathrm{cm}$ transverse incision above sternal notch should be done. The localised affected gland is then dissected and removed. The last step is comparison of measured ex vivo activity and neck activity after excision. When the value is over $20 \%$, it is an indication for surgery termination. The risk of occurrence of possible complications is very low and the overall cost of this technique is also lower in comparison to more invasive methods. Other advantages may be distinguished, such as short time of patient recovery, short operative time, and shorter hospitalisation [43]. The gamma probe-assisted procedure is also very useful in the excision of an ectopic parathyroid adenoma located within the thymus [46].

\section{Endoscopic parathyroidectomy}

Endoscopic surgeries are now the most common type of procedure worldwide due to their many advantages. Because it is used for the operation of parathyroid glands, it leaves the best cosmetic result offering a good and safe approach to deep and ectopic locations. The proper magnification should be used within this method so that it decreases the risk of intra-operative injury. Nevertheless, the general anaesthesia must be used, and the operating time is longer. Moreover, this method may be successfully used in complicated cases, such as retroesophageal parathyroid adenoma coexisting with an aberrant right subclavian artery [47]. During endoscopic parathyroidectomy the patient needs to be placed in a supine position. The neck remains positioned neutrally. After general anaesthesia is introduced, a $5 \mathrm{~mm}$ incision has to be made above the sternal notch followed by carbon monoxide insufflation to $10 \mathrm{mmHg}$. Using a $5 \mathrm{~mm} 0^{\circ}$ endoscope, dissection of the subplatysmal plane towards the side of the localised adenoma is performed followed by usage of a $30^{\circ}$ endoscope in order to place three ports in the lateral side of the neck. The dissection is performed laterally to the strap muscles and medially to the carotid artery until meeting the gland and identifying the recurrent laryngeal nerve. After the dissection of the gland, the ligation of the vascular pedicle with an ENDOLOOP is performed followed by its removal in a bag through the $5 \mathrm{~mm}$ port. Closure of the wound is possible by using Steri-Strips [41].

\section{Surgical approach to ectopic mediastinal parathyroid adenoma}

Ectopic adenomas are not located next to the thyroid gland and can cause problems with its localisation, even 
for experienced surgeons. The occurrence of ectopic adenomas is quite rare and occur in 3-4\% of all parathyroid adenoma cases [49]. Primary hyperparathyroidism caused by an ectopic mediastinal parathyroid adenoma is not very common either. Most abnormal parathyroid glands are localised in the superior mediastinum and can be removed by a cervical incision. However, there are cases when those glands are hard or impossible to access using traditional methods such as median sternotomy or thoracotomy [50]. Over the last decade, we saw significant technical advancements in detection and resection of affected glands using video-assisted thoracoscopic surgery (VATS). It is a modern alternative to median sternotomy, which was used for many years in cases where adenoma was not accessible through the neck. Video-assisted thoracoscopic surgery may be performed with subxiphoid and lateral thoracic approach. The latest studies showed that single-incision mediastinal tumour resection by subxiphoid access is a useful endoscopic surgical approach for the treatment of mediastinal ectopic parathyroid adenomas. The post-thoracotomy pain syndrome is limited, and the aesthetic outcomes are protreptic [51]. Most patients undergoing VATS do not suffer from serious complications, but some may require conversion to median sternotomy for thymoma removal in cases of false positive pre-operative imaging. However, with the appropriate radiological preparation before the procedure, VATS is an effective and safe approach to ectopic mediastinal parathyroid adenoma [52]. Moreover, this method may be used in complicated cases, such as retroesophageal parathyroid adenoma coexisting with an aberrant right subclavian artery. This approach is also useful when the parathyroid glands are in the posterior mediastinum or among adipose tissue very close to the diaphragm. Unfortunately, surgeons dealing with the treatment of primary hyperparathyroidism rarely have skills in thoracic surgery techniques, and, conversely, thoracic surgeons have exceptional contact with these patients. All this affects the ability to search for parathyroid glands and has a direct relationship to the results of surgery.

In preparation for VATS, it is worth performing Tc-99m sestamibi scintigraphy and endobronchial ultrasound biopsy. Because $\mathrm{Tc}(99)$ sestamibi reveals the localisation of an intense intake, endobronchial ultrasound-guided transbronchial needle aspiration (EBUS-TBNA) can be essential for diagnosis confirmation [53]. After surgery, it is still essential to measure the blood serum calcium levels because hypocalcaemia is seen with increasing frequency and occurs in about $30 \%$ of cases [54]. In such cases it requires oral supplementation with calcium, and in several cases together with activated vitamin D [56]. Patients who were suffering from hypercalcaemia and high PTH levels before sur- gery are at higher risk of transient hypoparathyroidism because of port-operative transient low blood serum calcium levels resulting from suppression of remaining parathyroid glands. Permanent hypercalcaemia also occurs as an outcome of removal and devascularisation of functioning glands but is quite rare [57]. The resection of parathyroid glands is the best therapy for PHPT. It has important outcomes such as correction of biochemical values, improvement of life quality, and lowering the bone fracture risk $[55,58]$.

\section{Conclusion}

Primary hyperparathyroidism is one of the main causes of hypercalcaemia in patients, who are usually diagnosed before clinically significant symptoms develop due to blood tests performed as preventive care or in order to diagnose some other disorders. Nevertheless, classical symptoms are related to abnormal function of parathyroid glands, resulting in elevated blood calcium levels, leading to disturbances of psychological functioning as well to disorders regarding urinary tract, and the gastrointestinal and central nervous system. The largest group of patients diagnosed with the PHPT are those around 55 years of age. The diagnosis is mainly based on measuring the level of calcium and PTH in blood, giving a guidepost to perform surgery, which, if there are no contraindications, is the most effective form of therapy with very low consumption of time and risk of recurrence.

\section{References}

1. Longo DL, Fauci AS, Kasper DL, Hausser SL, Jameson J, Loscalzo J. (eds). Harrison's: Principles of Internal Medicine. 18th ed. McGraw-Hill, New York 2012.

2. Gopinath P, Mihai R. Hyperparathyroidism. Surgery (Oxford). 2011; 29(9): 451-458, doi: 10.1016/j.mpsur.2011.06.015.

3. Cordellat IM. Hyperparathyroidism: primary or secondary disease? Reumatol Clin. 2012; 8(5): 287-291, doi: 10.1016/j.reuma.2011.06.001, indexed in Pubmed: 22089066

4. Colaço SM, Si M, Reiff E, et al. Hyperparathyroidism after radioactive iodine therapy. Am J Surg. 2007; 194(3): 323-327, doi: 10.1016/j.amjsurg.2007.04.005, indexed in Pubmed: 17693276.

5. Madkhali T, Alhefdhi A, Chen H, et al. Primary hyperparathyroidism. Ulus Cerrahi Derg. 2016; 32(1): 58-66, doi: 10.5152/UCD.2015.3032, indexed in Pubmed: 26985167.

6. Bandeira F, Griz L, Chaves N, et al. Sociedade Brasileira de Endocrinologia e Metabologia. Diagnosis and management of primary hyperparathyroidism - a scientific statement from the Department of Bone Metabolism, the Brazilian Society for Endocrinology and Metabolism. Arq Bras Endocrinol Metabol. 2013; 57(6): 406-424, doi: 10.1590/s0004-27302013000600002, indexed in Pubmed: 24030180.

7. Calvi LM, Sims NA, Hunzelman JL, et al. Activated parathyroid hormone/parathyroid hormone-related protein receptor in osteoblastic cells differentially affects cortical and trabecular bone. J Clin Invest. 2001; 107(3): 277-286, doi: 10.1172/JCI11296, indexed in Pubmed: 11160151.

8. Silverberg SJ, Lewiecki EM, Mosekilde L, et al. Diagnosis of asymptomatic primary hyperparathyroidism: proceedings of the third international workshop. J Clin Endocrinol Metab. 2009; 94(2): 340-350, doi: 10.1210/jc.2008-1758, indexed in Pubmed: 19193909.

9. Reiher AE, Mazeh H, Schaefer S, et al. Symptoms of gastroesophageal reflux disease improve after parathyroidectomy. Surgery. 2012; 152(6): 1232-1237, doi: 10.1016/j.surg.2012.08.051, indexed in Pubmed: 23158189. 
10. Gurrado A, Piccinni G, Lissidini G, et al. Hypercalcaemic crisis due to primary hyperparathyroidism - a systematic literature review and case report. Endokrynol Pol. 2012; 63(6): 494-502, indexed in Pubmed: 23339009

11. Jacobs TP, Bilezikian JP. Clinical review: Rare causes of hypercalcemia. JClin Endocrinol Metab. 2005; 90(11): 6316-6322, doi: 10.1210/jc.2005-0675, indexed in Pubmed: 16131579.

12. Reiher AE, Mazeh H, Schaefer S, et al. Symptoms of gastroesophageal reflux disease improve after parathyroidectomy. Surgery. 2012; 152(6): 1232-1237, doi: 10.1016/j.surg.2012.08.051, indexed in Pubmed: 23158189

13. Hinnie J. The management of primary hyperparathyroidism. Scott Med J. 2013; 58(4): 251-253, doi: 10.1177/0036933013507845, indexed in Pubmed: 24215047.

14. Tordjman KM, Greenman $Y$, Osher E, et al. Characterization of normocalcemic primary hyperparathyroidism. Am J Med. 2004; 117(11): 861-863, doi: 10.1016/j.amjmed.2004.06.037, indexed in Pubmed: 15589491.

15. Segiet OA, Deska M, Michalski M, et al. Molecular profiling in primary hyperparathyroidism. Head Neck. 2015; 37(2): 299-307, doi: 10.1002/hed.23656, indexed in Pubmed: 24595969.

16. Marini F, Cianferotti L, Giusti F, et al. Molecular genetics in primary hyperparathyroidism: the role of genetic tests in differential diagnosis, disease prevention strategy, and therapeutic planning. A 2017 update Clin Cases Miner Bone Metab. 2017; 14(1): 60-70, doi: 10.11138/cc$\mathrm{mbm} / 2017.14 .1 .060$, indexed in Pubmed: 28740527.

17. Zhao L, Sun Lh, Liu Dm, et al. Copy number variation in CCND1 gene is implicated in the pathogenesis of sporadic parathyroid carcinoma. World J Surg. 2014; 38(7): 1730-1737, doi: 10.1007/s00268-014-2455-9, indexed in Pubmed: 24510244.

18. Sulaiman L, Nilsson IL, Juhlin CC, et al. Genetic characterization of large parathyroid adenomas. Endocr Relat Cancer. 2012; 19(3): 389-407, doi: 10.1530/ERC-11-0140, indexed in Pubmed: 22454399.

19. Duan K, Gomez Hernandez K, Mete O. Clinicopathological correlates of hyperparathyroidism. J Clin Pathol. 2015; 68(10): 771-787 doi: 10.1136/jclinpath-2015-203186, indexed in Pubmed: 26163537.

20. Cavaco BM, Torrinha F, Mendonça E, et al. Preoperative diagnosis of suspicious parathyroid adenomas by RT-PCR using mRNA extracted from leftover cells in a needle used for ultrasonically guided fine needle aspiration cytology. Acta Cytol. 2003; 47(1): 5-12, doi: 10.1159/000326468, indexed in Pubmed: 12585024.

21. Kutahyalioglu M, Nguyen HaT, Kwatampora L, et al. Genetic profiling as a clinical tool in advanced parathyroid carcinoma. J Cancer Res Clin Oncol. 2019; 145(8): 1977-1986, doi: 10.1007/s00432-019-02945-9, indexed in Pubmed: 31309300.

22. Costa-Guda J, Imanishi Y, Palanisamy N, et al. Allelic imbalance in sporadic parathyroid carcinoma and evidence for its de novo origins. Endocrine. 2013; 44(2): 489-495, doi: 10.1007/s12020-013-9903-4, indexed in Pubmed: 23435613.

23. Sisson JC, Giordano TJ, Raymond VM, et al. First description of parathyroid disease in multiple endocrine neoplasia $2 \mathrm{~A}$ syndrome. Endocr Pathol. 2008; 19(4): 289-293, doi: 10.1007/s12022-008-9049-8, indexed in Pubmed: 19034701.

24. Deska M, Romuk E, Segiet OA, et al. Expression of TRAIL and Fas in Primary Hyperparathyroidism. J Invest Surg. 2017; 30(4): 242-246, doi: 10.1080/08941939.2016.1236159, indexed in Pubmed: 27763797.

25. Gawrychowski J, Kowalski G, Buła G. Parathyroid Carcinoma - Diagnosis and Surgical Treatment a 24-year Experience. Polish Journal of Surgery. 2010; 82(2), doi: 10.2478/v10035-010-0014-0.

26. Kelly KI, Chen H, Sippel RS. Primary hyperparathyroidism. Cance Treat Res. 2010; 153: 87-103, doi: 10.1007/978-1-4419-0857-5 6, indexed in Pubmed: 19957221.

27. Bradley SJ, Knodle KF. Ultrasound based focused neck exploration for primary hyperparathyroidism. Am J Surg. 2017; 213(3): 452-455, doi: 10.1016/j.amjsurg.2016.11.025, indexed in Pubmed: 27939023.

28. Kushchayeva YS, Tella SH, Kushchayev SV, et al. Comparison of hyperparathyroidism types and utility of dual radiopharmaceutical acquisition with Tc99m sestamibi and I for localization of rapid washout parathyroid adenomas. Osteoporos Int. 2019; 30(5): 1051-1057, doi: 10.1007/s00198-019-04846-6, indexed in Pubmed: 30706095

29. Paillahueque G, Massardo T, Barberán M, et al. [False negative spect parathyroid scintigraphy with sestamibi in patients with primary hyperparathyroidism]. Rev Med Chil. 2017; 145(8): 1021-1027, doi: 10.4067/s0034-98872017000801021, indexed in Pubmed: 29189860.

30. Johnson NA, Tublin ME, Ogilvie JB. Parathyroid imaging: technique and role in the preoperative evaluation of primary hyperparathyroidism. AJR Am J Roentgenol. 2007; 188(6): 1706-1715, doi: 10.2214/AJR.06.0938, indexed in Pubmed: 17515397.

31. Takumi K, Fukukura Y, Hakamada H, et al. CT features of parathyroid carcinomas: comparison with benign parathyroid lesions. Jpn J Radiol. 2019; 37(5): 380-389, doi: 10.1007/s11604-019-00825-3, indexed in Pubmed: 30863971.

32. Goroshi M, Lila AR, Jadhav SS, et al. Percentage arterial enhancement: An objective index for accurate identification of parathyroid adenoma/hy- perplasia in primary hyperparathyroidism. Clin Endocrinol (Oxf). 2017; 87(6): 791-798, doi: 10.1111/cen.13406, indexed in Pubmed: 28656592.

33. Schweighofer-Zwink G, Hehenwarter L, Rendl G, et al. [Imaging of parathyroid adenomas with F-18 choline PET-CT]. Wien Med Wochenschr. 2019; 169(1-2): 15-24, doi: 10.1007/s10354-018-0660-0, indexed in Pubmed: 30264384.

34. Keidar Z, Solomonov E, Karry R, et al. Preoperative [Tc]MIBI SPECT/CT Interpretation Criteria for Localization of Parathyroid Adenomas-Correlation with Surgical Findings. Mol Imaging Biol. 2017; 19(2): 265-270, doi: 10.1007/s11307-016-1013-2, indexed in Pubmed: 27704321.

35. Xue J, Liu Y, Ji T, et al. Comparison between technetium-99m methoxyisobutylisonitrile scintigraphy and ultrasound in the diagnosis of parathyroid adenoma and parathyroid hyperplasia. Nucl Med Commun. 2018; 39(12): 1129-1137, doi: 10.1097/MNM.0000000000000921, indexed in Pubmed: 30239472

36. Gawrychowski J, Buła G. Imaging diagnostics for primary hyperparathyroidism. Endokrynol Pol. 2013; 64(5): 404-408, doi: 10.5603/EP.2013.0024, indexed in Pubmed: 24186598

37. Dijkstra B, Healy C, Kelly LM, et al. Parathyroid localisation - current practice. J R Coll Surg Edinb. 2002; 47(4): 599-607, indexed in Pubmed: 12365423.

38. Bilezikian JP, Khan AA, Potts JT, et al. Third International Workshop on the Management of Asymptomatic Primary Hyperthyroidism. Guidelines for the management of asymptomatic primary hyperparathyroidism: summary statement from the third international workshop. J Clin Endocrinol Metab. 2009; 94(2): 335-339, doi: 10.1210/jc.2008-1763, indexed in Pubmed: 19193908.

39. Díaz-Aguirregoitia FJ, Emparan C, Gaztambide S, et al. Intraoperative monitoring of kinetic total serum calcium levels in primary hyperparathyroidism surgery. J Am Coll Surg. 2004; 198(4): 519-524, doi: 10.1016/j. jamcollsurg.2003.12.006, indexed in Pubmed: 15050999.

40. Bergenfelz A, Lindblom P, Tibblin S, et al. Unilateral versus bilateral neck exploration for primary hyperparathyroidism: a prospective randomized controlled trial. Ann Surg. 2002; 236(5): 543-551, doi: 10.1097/00000658200211000-00001, indexed in Pubmed: 12409657.

41. Inabnet WB, Dakin GF, Haber RS, et al. Targeted parathyroidectomy in the era of intraoperative parathormone monitoring. World J Surg. 2002; 26(8): 921-925, doi: 10.1007/s00268-002-6619-7, indexed in Pubmed: 12016471

42. Ryan JA, Lee FT. Maximizing outcomes while minimizing exploration in hyperparathyroidism using localization tests. Arch Surg. 2004; 139(8): 838-42; discussion 842, doi: 10.1001/archsurg.139.8.838, indexed in Pubmed: 15302692

43. Goldstein RE, Billheimer D, Martin WH, et al. Sestamibi scanning and minimally invasive radioguided parathyroidectomy without intraoperative parathyroid hormone measurement. Ann Surg. 2003; 237(5): 722-30; discussion 730, doi: 10.1097/01.SLA.0000064362.58751.59, indexed in Pubmed: 12724639.

44. Haldar A, Thapar A, Khan S, et al. Day-case minimally invasive excision of a giant mediastinal parathyroid adenoma. Ann R Coll Surg Engl. 2014; 96(5): e21-e23, doi: 10.1308/003588414X13946184900480, indexed in Pubmed: 24992407.

45. Kitada M, Yasuda S, Nana T, et al. Surgical treatment for mediastinal parathyroid adenoma causing primary hyperparathyroidism. J Cardiothorac Surg. 2016; 11: 44, doi: 10.1186/s13019-016-0461-8, indexed in Pubmed: 27056365

46. Daliakopoulos SI, Chatzoulis G, Lampridis S, et al. Gamma probe-assisted excision of an ectopic parathyroid adenoma located within the thymus: case report and review of the literature. J Cardiothorac Surg. 2014; 9: 62, doi: 10.1186/1749-8090-9-62, indexed in Pubmed: 24685256.

47. Nakada T, Akiba T, Inagaki T, et al. A case of a retroesophageal parathyroid adenoma with an aberrant right subclavian artery: a potential surgical pitfall. Ann Thorac Cardiovasc Surg. 2014; 20 Suppl: 786-789, doi: 10.5761/atcs.cr.12.02124, indexed in Pubmed: 23411847.

48. Sadler GP, Dudley N. Surgery for the parathyroid glands. In: Moriris PJ Wood WC. ed. Oxford textbook of surgery. 2. Oxford University Press, Oxford 2000: 1129-1141.

49. Panchani R, Varma T, Goyal A, et al. A challenging case of an ectopic parathyroid adenoma. Indian J Endocrinol Metab. 2012; 16(Suppl 2): S408-S410, doi: 10.4103/2230-8210.104110, indexed in Pubmed: 23565445.

50. Kim YSu, Kim J, Shin S. Thoracoscopic removal of ectopic mediastinal parathyroid adenoma. Korean J Thorac Cardiovasc Surg. 2014; 47(3): 317-319, doi: 10.5090/kjtcs.2014.47.3.317, indexed in Pubmed: 25207237.

51. Nagano H, Suda T, Ishizawa H, et al. Video-assisted thoracoscopic surgery for ectopic mediastinal parathyroid tumor: subxiphoid and lateral thoracic approach. J Thorac Dis. 2019; 11(7): 2932-2938, doi: 10.21037/jtd.2019.07.35, indexed in Pubmed: 31463122

52. Isaacs KE, Belete S, Miller BJ, et al. Video-assisted thoracoscopic surgery for ectopic mediastinal parathyroid adenoma. BJS Open. 2019; 3(6): 743-749, doi: 10.1002/bjs5.50207, indexed in Pubmed: 31832580.

53. Buderi SI, Saleh HZ, Theologou T, et al. Endobronchial ultrasound-guided biopsy to diagnose large posterior mediastinal parathyroid adenoma 
prior to video-assisted thoracoscopic resection. BMJ Case Rep. 2014; 2014, doi: 10.1136/bcr-2013-200131, indexed in Pubmed: 24825549.

54. Brasier AR, Nussbaum SR. Hungry bone syndrome: clinical and biochemical predictors of its occurrence after parathyroid surgery. Am J Med. 1988; 84(4): 654-660, doi: 10.1016/0002-9343(88)90100-3, indexed in Pubmed: 3400660.

55. Gawrychowski J, Kowalski G, Gawrychowska A. Parathyroid Cancer - Occurrence, Diagnosis, Treatment. Pol J Surg. 2008; 80(9), doi: 10.2478/v10035-008-0072-8

56. Gopinath P, Sadler GP, Mihai R. Persistent symptomatic improvement in the majority of patients undergoing parathyroidectomy for primary hyperparathyroidism. Langenbecks Arch Surg. 2010; 395(7): 941-946, doi: 10.1007/s00423-010-0689-z, indexed in Pubmed: 20658300.

57. Schneider DF, Mazeh H, Sippel RS, et al. Is minimally invasive parathyroidectomy associated with greater recurrence compared to bilateral exploration? Analysis of more than 1,000 cases. Surgery. 2012; 152(6): 1008-1015, doi: 10.1016/j.surg.2012.08.022, indexed in Pubmed: 23063313.

58. Hughes DT, Miller BS, Park PB, et al. Factors in conversion from minimally invasive parathyroidectomy to bilateral parathyroid exploration for primary hyperparathyroidism. Surgery. 2013; 154(6): 1428-34; discussion 1434, doi: 10.1016/j.surg.2013.04.020, indexed in Pubmed: 24008086.

59. Gupta M, Singhal L, Kumar A. Hyperparathyroidism Mimicking Metastatic Bone Disease: A Case Report and Review of Literature. J Adolesc Young Adult Oncol. 2018; 7(3): 400-403, doi: 10.1089/jayao.2017.0114, indexed in Pubmed: 29446693.
60. Allen E, Fingeret A. Anatomy. Head and Neck, Thyroid. StatPearls Publishing, Treasure Island (FL) 2019.

61. Zhu R, Wang Z, Hu Ya. Prognostic role of parafibromin staining and CDC73 mutation in patients with parathyroid carcinoma: A systematic review and meta-analysis based on individual patient data. Clin Endocrinol (Oxf). 2020; 92(4): 295-302, doi: 10.1111/cen.14161, indexed in Pubmed: 31945198.

62. Erickson LA, Mete O. Immunohistochemistry in Diagnostic Parathyroid Pathology. Endocr Pathol. 2018; 29(2): 113-129, doi: 10.1007/s12022-018-9527-6, indexed in Pubmed: 29626276.

63. Broos WAM, van der Zant FM, Knol RJ, et al. Choline PET/CT in parathyroid imaging: a systematic review. Nucl Med Commun. 2019; 40(2): 96-105, doi: 10.1097/MNM.0000000000000952, indexed in Pubmed: 30444749.

64. Buła G, Truchanowski W, Koziołek H, et al. A follow-up study of patients with MEN syndromes - five case reports. Endokrynol Pol. 2018; 69(2): 163-167, doi: 10.5603/EP.2018.0020, indexed in Pubmed: 29952424.

65. Schweighofer-Zwink G, Hehenwarter L, Rendl G, et al. [Imaging of parathyroid adenomas with F-18 choline PET-CT]. Wien Med Wochenschr. 2019; 169(1-2): 15-24, doi: 10.1007/s10354-018-0660-0, indexed in Pubmed: 30264384

66. Tariq M, Alhefdhi A, Chen $\mathrm{H}$, et al. Primary Hyperparathyrodoism. Ulus Cerrahi Derg. 2016; 32(1): 60, doi: 10.5152/UCD.2015.3032, indexed in Pubmed: 26985167. 